\title{
Immune mechanisms and the role of oxidative stress in intrahepatic cholestasis of pregnancy
}

\author{
ALI OZLER ${ }^{1}$, DERYA UCMAK ${ }^{2}$, MEHMET SIDDIK EVSEN ${ }^{1}$, IBRAHIM KAPLAN ${ }^{3}$, BILAL ELBEY \\ MUSTAFA ARICA ${ }^{2}$, MUHSIN KAYA \\ ${ }^{1}$ Department of Obstetrics and Gynecology, Faculty of Medicine, Dicle University, Diyarbakir, Turkey \\ ${ }^{2}$ Department of Dermatology, Faculty of Medicine, Dicle University, Diyarbakir, Turkey \\ ${ }^{3}$ Department of Biochemistry, Faculty of Medicine, Dicle University, Diyarbakir, Turkey \\ ${ }^{4}$ Department of Immunology, Faculty of Medicine, Dicle University, Diyarbakir, Turkey \\ ${ }^{5}$ Department of Gastroenterology, Faculty of Medicine, Dicle University, Diyarbakir, Turkey
}

\begin{abstract}
Introduction: The purpose of this study was to investigate levels of inflammatory cytokines such as interleukin $6(I L-6)$, tumor necrosis factor $\alpha(T N F-\alpha)$ and neopterin and oxidative stress status in patients with intrahepatic cholestasis of pregnancy (ICP) as well as their potential role in the pathophysiology.

Material and methods: Thirty patients with ICP (Group 1) and 30 healthy pregnant women (Group 2) were included in this prospective case-control study. Levels of IL-6, TNF- $\alpha$ and neopterin were determined in both of the groups. Total anti-oxidant status (TAS) and total oxidative stress (TOS) levels were determined by means of a fully automated Erel method. Oxidative stress index (OSI) was calculated as the ratio of TOS to TAS.

Results: There was no significant difference between the groups in IL-6 and TNF- $\alpha$ levels ( $p=$ $=0.105$ and $p=0.722$, respectively). The mean neopterin level was significantly higher in Group 1 compared to Group 2 (2.34 \pm 0.77 and $1.57 \pm 0.38$, respectively, $p=0.001)$. In addition, TAS, TOS and OSI levels were significantly higher in Group 1 ( $p=0.004, p=0.001$ and $p=0.001$, respectively) compared to Group 2.

Discussion: Intrahepatic cholestasis of pregnancy is an inflammatory disorder in which maternal immune reaction may play a role. Interleukin 6 and $T N F-\alpha$, which are some of the markers of humoral reaction, act as an indicator of abnormal reaction rather than acute-phase reaction in ICP. Further clinical trials and supportive placental findings are needed on the role of cytokines in cellular and humoral immune reactions during the symptomatic period and delivery to better understand the role of immune mechanisms in the aetiology of ICP.
\end{abstract}

Key words: intrahepatic cholestasis of pregnancy, neopterin, inflammation, interleukin, oxidative stress.

(Centr Eur J Immunol 2014; 39 (2): 198-202)

\section{Introduction}

Intrahepatic cholestasis of pregnancy (ICP) is a benign liver disorder specific to pregnancy, which occurs in the late second or third trimester and spontaneously resolves following delivery. Its incidence varies between $0.1 \%$ and $10 \%$ depending on geographical location and ethnicity [1]. Although its exact aetiology is unknown, various genetic, hormonal, dietary and environmental factors are suggested as etiologic factors [2]. It is one of the top items on the agenda of obstetricians and dermatologists, due to an increased risk of maternal and foetal morbidity and mor- tality. Its unclear aetiopathogenesis keeps physicians interested in this disorder. Unlike other forms of pregnancy dermatoses, it has no primary skin lesions and is characterised by severe itch typically located in the palmoplantar region, which spreads over the extremities and trunk. The lesions may vary in duration and severity and have a wide spectrum from superficial excoriation to prurigo nodularis [3]. It has no specific laboratory test result, which might facilitate diagnosis; however, elevated levels of bile acids and aminotransferases are assumed to have particular significance in the absence of previous diagnosis of liver disease [4]. Patients with ICP are exposed to increased maternal

Correspondence: Assist Prof. Ali Ozler, Department of Obstetrics and Gynecology, Faculty of Medicine, Dicle University, Diyarbakir, Turkey, e-mail: draliozler@gmail.com 
and foetal risks such as preeclampsia, post-partum bleeding, preterm delivery, meconium passage into amniotic fluid, abnormal foetal cardiac activity and even foetal death [5]. There are studies in the literature investigating the causes of spontaneous abortion, preeclampsia, intrauterine growth restriction and ICP, which put emphasis on inflammatory mechanisms [6]. Inflammatory changes in the fetoplacental unit may affect the maternal and foetal outcomes. Interleukin 6 (IL-6) and tumour necrosis factor $\alpha$ (TNF- $\alpha$ ) are known to be proinflammatory cytokines produced by macrophages of the liver (Kupffer cells), which take part in the elimination of extraintestinal pathogens, creation of inflammatory events and development of rapid immune reactions $[7,8]$. Neopterin, produced only by activated macrophages and monocytes, is a specific and distinctive marker of inflammation. Elevated plasma levels of neopterin were reported in patients with viral infections, severe systemic infectious and various autoimmune diseases and pregnancy-induced hypertensive diseases [9, 10].

Increased oxidative stress index (OSI) is the inability of total anti-oxidant status (TAS) to compensate for increased levels of total oxidative stress (TOS), which results in a marked imbalance between production and elimination of free radicals [11]. Increased levels of TOS were reported in pregnant women with hypertensive diseases, which might affect the maternal and foetal outcomes [12]. The purpose of this study was to assess the levels of inflammatory cytokines such as IL-6, TNF- $\alpha$ and neopterin as well as TOS in patients with ICP.

\section{Material and methods}

After the Local Ethics Committee's approval and informed consent from patients were obtained, 30 pregnant women with ICP who consecutively presented to the Dicle University Clinics of Gynaecology and Obstetrics, Gastroenterology and Dermatology in January-July 2013 were included prospectively. The diagnosis of ICP was done based on the following criteria:

- occurrence of intrahepatic cholestasis in the second or third trimester and rapid relief of signs and symptoms following delivery,

- elevated serum levels of transaminases,

- pruritus, accompanied by skin symptoms or not,

- absence of obstructive hepatobiliary pathologies in the clinical examination and ultrasonography,

- a negative viral hepatitis panel (hepatitis A, B and C), and absence of drug use, which might cause elevated liver enzymes [13, 14].

Group 1 consisted of 30 patients with ICP, whereas group 2 consisted of 30 control subjects who were matched in respect of gestational weeks and had no systemic diseases, which could complicate the pregnancy. The patients and the control subjects were evaluated in terms of age, gravida, parity, number of living children, gestational weeks, hematologic and biochemical parameters and serologic panels of viral hepatitis. In addition, the patients were examined by means of abdominal ultrasonography to rule out abnormalities of the liver and biliary tract. Patients with allergic diseases, viral or non-viral hepatitis, dermatitis, chronic liver diseases, diseases which might cause obstruction of the biliary tract (cholelithiasis - choledocholithiasis), hypothyroidism, hyperthyroidism, hypertensive diseases which might affect the liver functions (preeclampsia, eclampsia and HELLP), chronic heart, kidney or lung diseases and/or pregnancy-induced acute fatty liver were excluded from the study.

Blood samples were collected from all participants prior to the treatment and were immediately centrifuged at $4000 \mathrm{rpm}$ and $+4^{\circ} \mathrm{C}$ for 10 minutes. Collected sera were taken into Eppendorf tubes. Then, samples were transferred on ice and kept at $-70^{\circ} \mathrm{C}$ in a deep freezer until the time of analysis. Serum levels of neopterin, IL-6 and TNF- $\alpha$ were calculated by means of enzyme-linked immunosorbent assay according to the manufacturer's instructions (Neopterin; DRG Diagnostics International, Inc., Mountainside, NJ, USA) (IL-6 and TNF- $\alpha$; DIA Source, Nivelles, Belgium). Intra and inter assay coefficients of variations ranged from $3 \%$ to $7 \%$ for neopterin, IL-6 and TNF- $\alpha$.

Total anti-oxidant status and TOS levels were determined by means of a fully automated Erel method [15, 16].

Oxidative stress index was calculated as the ratio of TOS to TAS. Height and weight measurements were made and body mass index (BMI) was calculated at the same time when venous blood samples were collected.

Statistical analyses were performed by SPSS (Statistical Package for Social Sciences) for Windows 15.0 (SPSS, Inc., Chicago, IL, USA) and Excel programs. Data were expressed as means and standard deviations for numeric variables. Distribution of numeric data was assessed by Kolmogorov-Smirnov test. As data did not distribute normally, Mann-Whitney $U$ test was employed for the comparisons. $P$ values smaller than 0.05 were considered statistically significant.

\section{Results}

Demographic and clinical characteristics of the study subjects are presented in Table 1 . When evaluated in terms of the mean age, BMI, gestational weeks and obstetrics data, there was no statistically significant difference between the groups in clinical characteristics. Liver function test results and LDH levels were significantly higher in the patient group (group 1) compared to the control group (group 2) $(p<0.001)$. Hematologic and biochemical parameters are demonstrated in Table 2.

There was no significant difference between the groups in IL-6 and TNF- $\alpha$ levels ( $p=0.105$ and $p=0.722$, respectively). The mean neopterin level was significantly high- 
Table 1. Clinical characteristics of patients with intrahepatic cholestasis of pregnancy (ICP) and healthy pregnant women

\begin{tabular}{lccc}
\hline & $\begin{array}{c}\text { Patient group } \\
(\boldsymbol{n}=\mathbf{3 0}) \\
\text { Mean } \pm \text { SD }\end{array}$ & $\begin{array}{c}\text { Control group } \\
(\boldsymbol{n}=\mathbf{3 0}) \\
\text { Mean } \pm \mathbf{S D}\end{array}$ & $\boldsymbol{P}$ values \\
\hline Age & $29.7 \pm 5.9$ & $29.9 \pm 5.01$ & 0.88 \\
\hline Gestational week & $32.8 \pm 3.14$ & $31.6 \pm 4.51$ & 0.52 \\
\hline Gravity & $3.2 \pm 1.9$ & $2.97 \pm 1.94$ & 0.67 \\
\hline Parity & $1.6 \pm 1.5$ & $1.8 \pm 1.58$ & 0.56 \\
\hline Living children & $1.5 \pm 1.6$ & $1.7 \pm 1.44$ & 0.46 \\
\hline Body mass index & $27.8 \pm 4.6$ & $27.9 \pm 4.9$ & 0.94 \\
\hline
\end{tabular}

er in group 1 compared to group $2(2.34 \pm 0.77$ and 1.57 \pm 0.38 , respectively, $p=0.001$ ). The mean IL- 6 and TNF- $\alpha$ levels are demonstrated in Table 3 . In addition, TAS, TOS and OSI levels were significantly higher in group 1 ( $p=$ $=0.004, p=0.001$ and $p=0.001$, respectively) compared to group 2 .

\section{Discussion}

Numerous factors have been held responsible for the pathogenesis of ICP. Although not a specific diagnostic criterion, the aminotransferase level has been reported to assume importance in the diagnosis of ICP. The foetus is an allogeneic material of the maternal body in which inflammatory events occur from the moment of implantation. Because of maternal immune system adaptation, the foetus is not perceived as a foreign body and immune mechanisms are not triggered in the maternal body [17]. Placental vascular insufficiencies and immune factors are

Table 3. Levels of pro-inflammatory cytokines and oxidative stress status in patient and the control groups

\begin{tabular}{lccc}
\hline & $\begin{array}{c}\text { Patient group } \\
(\boldsymbol{n}=\mathbf{3 0}) \\
\text { Mean } \pm \text { SD }\end{array}$ & $\begin{array}{c}\text { Control group } \\
(\boldsymbol{n}=\mathbf{3 0}) \\
\text { Mean } \pm \text { SD }\end{array}$ & $\boldsymbol{P}$ values \\
\hline $\mathrm{IL}-6(\mathrm{ng} / \mathrm{dl})$ & $3.51 \pm 2.69$ & $2.01 \pm 0.99$ & 0.105 \\
\hline TNF- $\alpha(\mathrm{ng} / \mathrm{dl})$ & $7.45 \pm 1.91$ & $7.26 \pm 2.32$ & 0.722 \\
\hline Neopterin $(\mathrm{nmol} / \mathrm{l})$ & $2.34 \pm 0.77$ & $1.57 \pm 0.38$ & $\mathbf{0 . 0 0 1}$ \\
\hline $\begin{array}{l}\text { TOS }\left(\mu \mathrm{M} \mathrm{H}_{2} \mathrm{O}_{2} \mathrm{Eq} . /\right. \\
\text { g protein })\end{array}$ & $101.9 \pm 43.4$ & $41.63 \pm 23.3$ & $\mathbf{0 . 0 0 1}$ \\
\hline $\begin{array}{l}\text { TAS } \\
(\mu \mathrm{M} \text { TroloxEq./ }\end{array}$ & $1.58 \pm 0.19$ & $1.45 \pm 0.13$ & $\mathbf{0 . 0 0 4}$ \\
g protein) & & & \\
\hline OSI & $65.2 \pm 27.7$ & $28.8 \pm 15.95$ & $\mathbf{0 . 0 0 1}$
\end{tabular}

$\overline{\text { TOS - total oxidative stress; TAS - total anti-oxidant status; OSI-oxidative }}$ stress index
Table 2. Complete blood count and biochemical parameters of the subjects in the patient and control groups

\begin{tabular}{lccc}
\hline & $\begin{array}{c}\text { Patient group }(\boldsymbol{n} \\
\boldsymbol{=} \mathbf{3 0}) \\
\text { Mean } \pm \text { SD }\end{array}$ & $\begin{array}{c}\text { Control group } \\
(\boldsymbol{n}=\mathbf{3 0}) \\
\text { Mean } \pm \text { SD }\end{array}$ & $\boldsymbol{P}$ values \\
\hline $\mathrm{Hb}(\mathrm{g} / \mathrm{dl})$ & $11.9 \pm 1.4$ & $11.8 \pm 1.17$ & 0.53 \\
\hline $\mathrm{Hct}(\%)$ & $35.5 \pm 3.9$ & $36.8 \pm 3.29$ & 0.16 \\
\hline $\mathrm{WBC}(\mathrm{K} / \mu \mathrm{l})$ & $9763 \pm 2067$ & $9720 \pm 2385$ & 0.94 \\
\hline TC $(\mathrm{K} / \mathrm{ll})$ & $258.2 \pm 82.9$ & $246.7 \pm 54.8$ & 0.95 \\
\hline MPV $(\mathrm{fl})$ & $9.43 \pm 1.4$ & $9.28 \pm 1.1$ & 0.63 \\
\hline AST $(\mathrm{U} / \mathrm{l})$ & $161.6 \pm 140.9$ & $12.8 \pm 4.1$ & $\mathbf{0 . 0 0 1}$ \\
\hline ALT $(\mathrm{U} / \mathrm{l})$ & $242.9 \pm 237.8$ & $14.9 \pm 6.1$ & $\mathbf{0 . 0 0 1}$ \\
\hline LDH $(\mathrm{mg} / \mathrm{dl})$ & $336.7 \pm 159.2$ & $226.1 \pm 56.5$ & $\mathbf{0 . 0 0 1}$
\end{tabular}

Hb-haemoglobin; Hct-haematocrit; WBC-white blood cells; $T C$ - thrombocyte count; $M P V$ - mean thrombocyte volume; $A S T$ - aspartate aminotransferase; $A L T$ - alanine aminotransferase; $L D H$ - lactate dehydrogenase

known to take part in the aetiology of some disorders including spontaneous abortion, hypertensive pregnancies and intrauterine growth retardation (IUGR). Recent studies on patients with ICP have also put emphasis on the placental-vascular and maternal inflammatory processes. Studies on ICP patients who had intrauterine foetal death or meconium aspiration reported reduced perfusion in these patients, caused by accumulation of bile acids in the vascular bed $[1,18]$. Interleukin 6 , TNF- $\alpha$ and neopterin, which are released by activated macrophages and monocytes in the body, are proinflammatory cytokines that play a role in the innate immune system [13]. It was shown that maternal-foetal autoimmune reactions might lead to miscarriages and complications $[19,20]$. Prospective case control studies reported that neopterin levels were significantly higher in hypertension-complicated pregnancies with adverse maternal and foetal outcomes [21-25].

Wang et al. were the first to investigate neopterin and soluble IL-2 receptors in patients with ICP [26]. They reported that plasma levels of neopterin and soluble IL-2 receptors were elevated in patients with ICP; however, this result was not associated with the early weeks of pregnancy or low birth weight. In the present study, it was found that plasma levels of neopterin were significantly higher in the ICP group compared to controls. There are only a limited number of studies in the literature investigating the immune mechanisms in patients with ICP. Shi et al. reported reduced levels of immunoglobulins and CD8 lymphocytes and an increased CD4/CD8 ratio in patients with ICP. In addition, they suggested that cellular immunity took part in the development of ICP [20].

Interleukin 6 and TNF- $\alpha$ are produced in both humoral and cellular immune reactions. The main tasks of these cytokines are activation of the acute-phase reaction, B-cell 
differentiation (immunoglobulin release), stimulation of growth in differentiated B-cells and activation of acutephase hepatic reaction [27]. To the best of our knowledge, this is the first study investigating IL-6 and TNF- $\alpha$ levels in patients with ICP. In this study, although higher levels of IL-6 and TNF- $\alpha$ were found in ICP patients compared to healthy controls, the difference between the groups did not reach a statistically significant level.

Placental ischemia has been suggested to be responsible for foetal hypoxia, meconium aspiration and even foetal death in patients with ICP. It assumes a prominent role in the increased oxidative stress. In the present study, TOS, TAS and OSI levels were found to be significantly higher in ICP patients compared to the controls. We believe that increased TOS and oxidative stress index was caused by the augmented inflammatory mechanisms in patients with ICP.

In conclusion, ICP may be an inflammatory disorder with liver and biliary tract involvement in which immune reaction plays a role. Although there is limited information in the literature, it was shown by this study that cellular immune reactions play a bigger role in this disorder than humoral reactions. Interleukin 6 and TNF- $\alpha$, which are some of the markers of the humoral reaction, act as an indicator of abnormal reaction rather than acute-phase reaction in ICP. In this respect, prospective clinical trials should be conducted on foetal cord blood taken from the patient both at the outset of the complaints and during delivery to better understand the role of cytokines in the cellular and humoral immune reactions in ICP. We believe that increased levels of oxidative stress, which cannot be compensated for by increased anti-oxidative mechanisms, may influence the maternal and foetal clinical consequences. Anti-oxidative treatment may be helpful in managing the increased inflammatory events and oxidative stress in patients with ICP; however, further clinical trials are needed to investigate the maternal and foetal outcomes of this disease.

\section{The authors declare no conflict of interest.}

\section{References}

1. Geenes V, Williamson C (2009): Intrahepatic cholestasis of pregnancy. World J Gastroenterol 15: 2049-2066.

2. Yamamoto Y, Moore R, Hess HA, et al. (2006): Estrogen receptor alpha mediates 17alpha-ethynylestradiol causing hepatotoxicity. J Biol Chem 281: 16625-16631.

3. Ambros-Rudolph CM (2006): Dermatoses of pregnancy. J Dtsch Dermatol Ges 9: 748-759.

4. Ambros-Rudolph CM, Glatz M, Trauner M, et al. (2007): The importance of serum bile acid level analysis and treatment with ursodeoxycholic acid in intrahepatic cholestasis of pregnancy: a case series from central Europe. Arch Dermatol 143: 757-762.

5. Kroumpouzos G (2002): Intrahepatic cholestasis of pregnancy: what's new. J Eur Acad Dermatol Venereol 16: 316-318.
6. Dong M, Shi Y (1996): Evaluation of cholic glycine in serum and hemorrheological parameters in women with intrahepatic cholestasis of pregnancy. Curr Adv Obstet Gynecol 5: 251253.

7. Dekker GA, Sibai BM (1999): The immunology of preeclampsia. Semin Perinatol 23: 24-33.

8. McClain CJ, Song Z, Barve SS, et al. (2004): Recent advances in alcoholic liver disease. IV. Dysregulated cytokine metabolism in alcoholic liver disease. Am J Physiol Gastrointest Liver Physiol 287: G497-502.

9. Rao RK, Seth A, Sheth P (2004): Recent advances in alcoholic liver disease: role of intestinal permeability and endotoxemia in alcoholic liver disease. Am J Physiol Gastroint Liver Physiol 286: G881-884.

10. Ozler A, Turgut A, Sak ME, et al. (2012): Serum levels of neopterin, tumor necrosis factor-alpha and Interleukin-6 in preeclampsia: relationship with disease severity. Eur Rev Med Pharmacol Sci 16: 1707-1712.

11. Evsen MS, Ozler A, Gocmez C, et al. (2013): Effects of estrogen, estrogen/progesteron combination and genistein treatments on oxidant/antioxidant status in the brain of ovariectomized rats. Eur Rev Med Pharmacol Sci 17: 1869-1873.

12. Vural P, Akgül C, Yildirim A, Canbaz M (2000): Antioxidant defence in recurrent abortion. Clin Chim Acta 295: 169-177.

13. Fuchs D, Weiss G, Wachter H (1993): Neopterin, biochemistry and clinical use as a marker for cellular immune reactions. Int Arch Allergy Immunol 101: 1-6.

14. Yılmaz S, Üstün Y, Hızlı D, Deveer R (2012): Gebeliğin İntrahepatik Kolestazi. Gazi Med J 23: 138-144.

15. Erel O (2004): A novel automated method to measure total antioxidant response against potent free radical reactions. Clin Biochem 37: 112-119.

16. Erel O (2005): A new automated colorimetric method for measuring total oxidant status. Clin Biochem 38: 1103-1111.

17. Johnson PM, Christmas SE, Vince GS (1999): Immunological aspects of implantation and implantation failure. Hum Reprod 14 Suppl 2: 26-36.

18. Sepúlveda WH, González C, Cruz MA, Rudolph MI (1991): Vasoconstrictive effect of bile acids on isolated human placental chorionic veins. Eur J Obstet Gynecol Reprod Biol 42: 211-215.

19. Dong M, Xie X, Wang Z, et al. (2002): Impaired mixed lymphocyte reaction in intrahepatic cholestasis of pregnancy. Gynecol Obstet Invest 54: 191-195.

20. Shi Q, Liu S, Xiong Q (1998): The changes of serum estrogen, progesterone and the function of immune system in intrahepatic cholestasis of pregnancy. Zhonghua Fu Chan Ke Za Zhi 33: 724-726.

21. Bichler A, Fuchs D, Hausen A, et al. (1983): Measurement of urinary neopterin in normal pregnant and non-pregnant women and in women with benign and malignant genital tract neoplasms. Arch Gynecol 233: 121-130.

22. Fuith LC, Fuchs D, Hausen A, et al. (1991): Neopterin, a marker of cell-mediated immune activation in human pregnancy. Int J Fertil 36: 372-375.

23. Haeger M, Unander M, Norder-Hansson B, et al. (1992): Complement, neutrophil, and macrophage activation in women with severe preeclampsia and the syndrome of hemolysis, elevated liver enzymes, and low platelet count. Obstet Gynecol 79: 19-26.

24. Kaleli I, Kaleli B, Demir M, et al. (2005). Serum levels of neopterin and interleukin-2 receptor in women with severe preeclampsia. J Clin Lab Anal 19: 36-39. 
25. Schröcksnadel H, Fuchs D, Herold M, et al. (1994): Activated macrophages in the pathologic mechanism of pregnancy-induced hypertension. Zentralbl Gynakol 116: 274-275.

26. Wang Z, Dong M, Chu H, He J (2004): Increased serum levels of neopterin and soluble interleukin-2 receptor in intrahepatic cholestasis of pregnancy. Acta Obstet Gynecol Scand 83: 1067-1070.

27. Licinio J, Kling MA, Hauser P (1998): Cytokines and brain function: relevance of interferon- $\alpha$ induced mood and cognitive changes. Semin Oncol 25: 30-38. 